\title{
CONFORTO TÉRMICO COM USO DE CLIMATIZAÇÃO POR ASPERSORES PARA VACAS GIROLANDO NA PRÉ - ORDENHA
}

GOMES, Ariel Feiria ${ }^{1}$

PEREIRA, Ariany Dos Reis ${ }^{1}$

BARBOSA, Mônica Machado ${ }^{1}$

MELLO, Silvio De Paula ${ }^{2}$

\author{
Recebido em: 2021.03.10 Aprovado em: $2021.05 .03 \quad$ ISSUE DOI: $10.3738 / 21751463.3904$
}

\begin{abstract}
Resumo: O termo estresse térmico é usado quando o calor gerado pelo animal somado ao calor absorvido do ambiente é maior que a capacidade deste em dissipá-la, esse fato influencia muito no resultado na produção de leite dos bovinos. O estresse térmico pode resultar em uma redução de $17 \%$ na produção de leite de vacas de produção de $15 \mathrm{~kg}$ leite/dia e em vacas de $40 \mathrm{~kg}$ leite/dia essa diminuição pode chegar até $22 \%$ na redução da produção. O objetivo deste trabalho foi avaliar o bem-estar dos animais e seus índices de conforto térmico, na ausência e presença de aspersores em vacas girolando na pré-ordenha no setor de Bovinocultura Leiteira da Faculdade Dr. Francisco Maeda- FAFRAM. Foram avaliadas 20 vacas girolando, entre os dias 10 de Fevereiro a 20 de Março no curral de espera. Foram coletadas informações referentes a condições climáticas (Temperatura de globo negro e umidade relativa do ar), o aspecto fisiológico (temperatura do pelame) que foram coletadas no curral de espera no sol, na sombra e com aspersores. As informações relativas às condições climáticas permitiram calcular o índice de temperatura de globo e umidade (ITGU). Concluiu-se que o uso de sombrites com aspersores melhora as condições para que os animais alcancem um conforto térmico, e consequentemente aumentem a produção.
\end{abstract}

Palavras-chave: Ambiente. Bem-estar. Temperatura

\section{THERMAL COMFORTH WITH THE USE OF CLIMATIZATION BY SPRAYERS FOR COWS TURNING IN PRE- MILKING}

SUMMARY: The term thermal stress is used when the heat generated by the animal added to the heat absorbed from the environment is greater than the capacity of the animal to dissipate it, this fact greatly influences the result in the milk production of cattle. Heat stress can result in a $17 \%$ drop in milk production from $15 \mathrm{~kg}$ milk / day cows and in $40 \mathrm{~kg}$ milk / day cows this value can reach $22 \%$. The objective of this work was to evaluate the welfare of the animals and their indexes of thermal comfort, in the absence and presence of sprinklers in rotating cows in premilking in the sector of Dairy Cattle of the Faculty Dr. Francisco Maeda - FAFRAM. Twenty gyrating cows were evaluated between the 10th of February and the 20th of March in the FAFRAM waiting corral. Information was collected regarding climatic conditions (black globe temperature and relative air humidity), physiological aspects (fur temperature) that were collected in the waiting pen in the sun, in the shade and with sprinklers. Through the environmental data, the globe temperature and humidity index (ITGU) was calculated. It was concluded that the use of shades with sprinklers improves the conditions for the animals to reach thermal comfort, and consequently increase the production.

Keywords: Sprinklers.Environment. Welfare. Temperature

\section{INTRODUÇÃO}

O Brasil se encontra entre os cinco maiores produtores de leite do mundo, com total de 34 Bilhões de litros de leite (CUNHA,2020).

Pelo fato do Brasil ser um país de clima tropical e subtropical, o estresse térmico pode surgir nos animais com mais facilidade, devido aos altos níveis de radiação solar e temperatura,

\footnotetext{
${ }^{1}$ Médico(a) Veterinário(a)

${ }^{2}$ Docente dos cursos de Agronomia e Medicina Veterinária Faculdade Dr. Francisco Maeda/Fundação Educacional de Ituverava.
}

Nucleus Animalium, v.13, n.1, maio 2021 
afetando dessa forma a produção. Em bovinos, o estresse térmico tem alto impacto na produtividade e, além disso, tem um alto impacto no desempenho reprodutivo das fêmeas também por serem sensíveis a altas temperaturas (ROSA, 2019).

Com relação a isso, temperaturas elevadas para vacas em lactação na pré-ordenha, podem atingir de $27^{\circ} \mathrm{C} 29^{\circ} \mathrm{C}$ respectivamente (BACCARI JUNIOR, 1998, ALMEIDA.,2009).

O termômetro globo negro (TGN), mede o índice de temperatura e umidade relativa do ar, em que são dados utilizados para avaliar o ambiente (MATARAZZO, 2004). Segundo Araújo et al., (2014), a faixa TGN para vacas em lactação de 7 a $26^{\circ} \mathrm{C}$ é considerada ótima, entre 27 e $34^{\circ} \mathrm{C}$ é considerada regular e acima de $35^{\circ} \mathrm{C}$ é considerada crítica.

Em relação ao índice de temperatura e umidade, pode ser calculado através da temperatura do bulbo seco e da umidade relativa do ar. Valores iguais a 70 ou menos, indica condição de conforto para os animais; valores entre 71 e 78 considera-se como valores que os animais já estão em estresse térmico; entre 79 e 83, indica perigo; acima de 83 pode ser considerado prejudicial à saúde do animal (BROWN-BRANDL, 2018).

O pelame serve como proteção contra, frio e calor promovendo manutenção da temperatura corporal, relacionada a trocas de calor com o ambiente (FERREIRA et al., 2017), Possui características como cor, que é associada aos mecanismos termorreguladores, a temperatura do pelame pode ser coletada de forma simples e rápida, utilizando um termômetro infravermelho digital (CONCEIÇÃO, 2008). Valores que estão entre $31,6^{\circ}$ e $34,7^{\circ} \mathrm{C}$ não mostram sofrimento por estresse térmico em bovinos nos ambientes climatizados.

A adaptação dos bovinos aos ambientes que são oferecidos a estes precisa ser verificada com cautela para que esses animais não sofram. São vários aspectos que abrangem esse estado de bem-estar, incluindo estado de saúde, condição física, fisiológica e emocional (CERQUEIRA, 2013).

Foi verificado em alguns estudos que a climatização do ambiente para o gado leiteiro (sistemas de resfriamento, nebulização e aspersão) quando associados a ventilação forçada, diminui a temperatura máxima do local, em relação ao ambiente externo, deve-se analisar o tipo de sistema a ser adotado, observar o custo benefício é seleção dos animais para cada região (DALTRO, 2018).

A aspersão direta de água nas vacas conduz o calor do corpo para o ambiente e os animais conseguem evaporar mais umidade da pele e permite que as vacas usem o resultante calor latente de evaporação para o resfriamento do corpo. Com esse sistema, as compensações induzidas pelo calor diminuem e vários outros fatores que causam a produção de leite menor, 
dessa forma a energia utilizada para o resfriamento do corpo pode ser poupada para a melhoria na produção. (DOMINGOS et al., 2012).

O objetivo deste trabalho foi avaliar o bem-estar dos animais e seus índices de conforto térmico, na ausência e presença de aspersores em vacas girolando na pré-ordenha no setor de Bovinocultura Leiteira da Faculdade Dr. Francisco Maeda- FAFRAM.

\section{MATERIAL E MÉTODO}

Aprovado pela Comissão de Ética no Uso de Animais da Faculdade Dr. Francisco Maeda (CEUA/FAFRAM), protocolo 44/2019.

O experimento foi realizado no setor de Bovinocultura Leiteira da Faculdade Dr. Francisco Maeda - FAFRAM, da Fundação Educacional de Ituverava- SP, localizada no Sítio das Acácias.

Foram avaliadas 20 vacas girolando, entre os dias 10 de Fevereiro a 20 de Março no curral de espera da FAFRAM.

Foram instalados canos de PVC e posteriormente aspersores em uma torneira e foi utilizado um sombrite por cima desses canos.

Foram coletadas informações referentes a condições climáticas (Temperatura de globo negro e umidade relativa do ar), os aspectos fisiológicos (temperatura do pelame) que foram coletadas no curral de espera no sol, na sombra e com aspersores.

Através dos dados ambientais foi calculado o índice de temperatura de globo e umidade (ITGU).

\section{ITGU: Tg + 0,36 Tpo + 41,5}

$\mathrm{Tg}=$ temperatura do termômetro de globo negro, ${ }^{\circ} \mathrm{C}$;

Tpo $=$ temperatura do ponto de orvalho, ${ }^{\circ} \mathrm{C}$.

As coletas foram realizadas, uma vez por semana, em dois horários distintos, pela manhã (07:00h) e a tarde (14:00h).

A temperatura do pelame foi coletada por um termômetro infra-vermelho e os dados climáticos foram coletados por um termohigrometro de bulbo seco e bulbo úmido e globo negro.

Os dados foram submetidos à análise de variância e as médias comparadas pelo teste de Tukey, a 5\% de probabilidade.

\section{RESULTADO E DISCUSSÃO}

Nas tabelas abaixo (1 e 2), estão descritas as médias da umidade relativa (UR\%), temperatura do ambiente (TA), temperatura do globo negro (TGN), índice de temperatura de 
globo e umidade (ITGU) e a temperatura do pelame (TP) de vacas girolando no curral de espera com o uso de sombreamento, expostas ao sol e com uso de aspersores, conforme os horários descritos nas Tabelas 1 e 2.

Tabela 1 - Médias da temperatura do pelame (TP), umidade relativa do ar (UR), temperatura do ambiente (TA) e de globo negro (TGN) e índice de temperatura de globo e umidade (ITGU) de vacas girolando no curral de espera de acordo com o sombreamento e aspersão. Ituverava/SP, 2020.

\begin{tabular}{|c|c|c|c|c|c|}
\hline Sombreamento & $\mathrm{UR} \%$ & $\mathrm{TA}\left({ }^{\circ} \mathrm{C}\right)$ & TGN $\left({ }^{\circ} \mathrm{C}\right)$ & ITGU & $\mathrm{TP}\left({ }^{\circ} \mathrm{C}\right)$ \\
\hline Sem Sombrite & $55,44^{\mathrm{a}}$ & $27,85 \mathrm{a}$ & $36,3 a$ & 84,3 & $38,69 \mathrm{a}$ \\
\hline Com Sombrite & $57,29^{a}$ & $29,15 \mathrm{a}$ & $35,3 \mathrm{a}$ & 83,89 & $38,04 b$ \\
\hline Sombrite/ Aspersor & $60,91 b$ & $26,1 b$ & $29,18 b$ & 77,4 & $38,9 \mathrm{a}$ \\
\hline Média Geral & 57,88 & 27,7 & 33,59 & 81,86 & 38,54 \\
\hline $\mathrm{CV} \%$ & 4,8 & 5,52 & 11,46 & 4,73 & 1,17 \\
\hline
\end{tabular}

Foi avaliado que as vacas que estavam no curral de espera expostas ao sol e com uso de sombrite e aspersores apresentaram diferenças significativas.

As variáveis apresentaram altos valores quando os animais estavam expostos ao sol $\left(\mathrm{UR}=55,44 \%, \quad \mathrm{TA}=27,85^{\circ} \mathrm{C}, \quad \mathrm{TGN}=36,3^{\circ} \quad \mathrm{C}, \quad \mathrm{ITGU}=84,3\right.$ e $\left.\mathrm{TP}=38,69^{\circ} \mathrm{C}\right)$, visivelmente identificando um desconforto térmico nos animais. Segundo Araújo (2018), animais de origem zebuína, indica que a zona de termoneutralidade é até a temperatura de $35^{\circ} \mathrm{C}$.

Foi verificado em alguns estudos que os valores de ITGU até 74 para bovinos definem situação de conforto; de 75 a 78 significam situação de alerta; de 79 a 84, perigo; e acima de 84, emergência (BAÊTA. SOUZA, 2010).

Foi verificado nos resultados acima que houve uma diferença significativa em TA quando foi inserido sombrite e aspersor no ambiente e segundo o resultado de Silva (2002) não houve diferença nas variáveis ambientais em seu trabalho, sendo assim, não apresentando diferença significativa. 
Tabela 2 - Médias da temperatura do pelame (TP), umidade relativa do ar (UR), temperatura do ambiente (TA) e de globo negro (TGN) e índice de temperatura de globo e umidade (ITGU) de vacas girolando no curral de espera de acordo com o horário. Ituverava/SP, 2020.

\begin{tabular}{llllll}
\hline Horário & UR\% & TA $\left({ }^{\circ} \mathrm{C}\right)$ & TGN $\left({ }^{\circ} \mathrm{C}\right)$ & ITGU & TP $\left({ }^{\circ} \mathrm{C}\right)$ \\
\hline $7: 00 \mathrm{~h}$ & $60,94^{\mathrm{a}}$ & $25,38 \mathrm{a}$ & $31,01 \mathrm{a}$ & $78,75^{\mathrm{a}}$ & $37,57 \mathrm{a}$ \\
14:00h & $56,4 \mathrm{~b}$ & $30,03 \mathrm{~b}$ & $36,18 \mathrm{~b}$ & $84,98 \mathrm{~b}$ & $38,82 \mathrm{~b}$ \\
\hline Média Geral & 58,67 & 27,7 & 33,6 & 81,86 & 38,19 \\
CV\% & 3,87 & 8,38 & 7,68 & 3,8 & 1,62 \\
\hline
\end{tabular}

Médias seguidas da mesma letra, na coluna, não diferem estatisticamente pelo teste de Tukey $(\mathrm{P}>0,05)$

Fonte: Elaborado pelo autor (2020).

Como observado na Tabela 2, as características avaliadas no curral de espera nos horários das 07:00h e 14:00h teve uma diferença significativa, em que foi visto que na parte da manhã obteve-se números que mostram que estão mais perto do conforto térmico dos animais, com números menores (UR - 60,94\%, TA $-25,38^{\circ} \mathrm{C}$, TGN $-31,01^{\circ} \mathrm{C}$, ITGU $-78,75, \mathrm{TP}-37,57^{\circ} \mathrm{C}$ ). Segundo estudos realizados a umidade relativa tem um intervalo considerado ideal para bovinos leiteiros de 50 a $70 \%$ e foi verificado no presente estudo que a umidade se apresentou dentro desse intervalo (BRETTAS, 2017). A umidade do ar é essencial na dissipação de calor pelo organismo do bovino, em que um ambiente muito quente e muito úmido pode prejudicar as perdas evaporativas em que são mais eficazes em locais quentes e secos (BOHMANOVA; MISZTAL; COLE, 2007).

Alguns estudos verificaram que animais expostos diretamente ao sol, quando receberam água sob aspersão, obtiveram maiores valores do teor de gordura e de proteína do leite. Os animais que ficaram na sombra tiveram o aumento no teor de gordura e de proteína quando a aspersão não ocorreu (BARBOSA et al., 2004). O estresse térmico pode ser controlado pelo manejo e adoção de práticas que favorecem a redução deste, como construção de sombras e fornecimento de água com fácil acesso (VITOR ; BITTAR, 2018). Para Daltro (2018), os bovinos estão suscetíveis ao estresse calórico quando a umidade relativa e a temperatura ambiente ultrapassam a zona de conforto, em que pode dificultar a dissipação de calor, aumentando assim a temperatura corporal e causando perdas na produção. Segundo o trabalho de Almeida, (2013), a umidade relativa do ar esteve mais elevada no turno da manhã e analisando os resultados do presente trabalho, foi visto também que no turno da manhã a UR estava com valor mais elevado em relação ao período da tarde. 


\section{CONCLUSÃO}

Concluiu-se que o uso de sombrites com aspersores melhora as condições para que os animais alcancem um conforto térmico, e consequentemente aumentem a produção.

\section{REFERÊNCIAS}

ALMEIDA, G.L.P.Climatização na pré-ordenha de vacas da raça girolando e seus efeitos na produção e qualidade do leite e no comportamento animal. 2009. 134 f. Dissertação. (Mestrado) - Curso de Engenharia Agronômica, Experimental, Universidade Federal do Pernambuco, Recife, 2009. Cap. 4.

ARAÚJO, P. M. Influência do Tipo de Ordenha e Temperamento de Bovinos da Raça Gir e Bubalinos da Raça Murrah sobre Produção e Composição do Leite. 115f. Tese (Doutorado em Psicobiologia), Experimental. Programa de Pós-Graduação em Psicobiologia. Universidade Federal do Rio Grande do Norte, Natal, 2018.

ARAUJO, J. I. M. et al. Efeitos das Variáveis Climáticas Sobre Características Fisiológicas de Vacas Mestiças (HOLANDÊS X GIR) Em Lactação. Revista Acadêmica Ciência Animal, Bom Jesus-Pi, v. 12, n. 8, p. 1-9, 18 ago. 2014. Semestral.

BROWN-BRANDL, Tami M. Understanding heat stress in beef cattle. Revista Brasileira de Zootecnia, [S.L.], v. 47, p. 1-2, 29 nov. 2018. Semestral. Fap UNIFESP (SCIELO). http://dx.doi.org/10.1590/rbz4720160414..

BACCARI ,Jr., F. Adaptação de Sistemas de Manejo na Produção de Leite em Clima Quente. In: Simpósio Brasileiro de Ambiência na Produção de Leite, Piracicaba, 1998. Anais... Piracicaba: FEALQ, 1999. p.24-67.

BAÊTA, F. C.; SOUZA, C. F. Ambiência em Edificações Rurais: Conforto animal. 2. ed. Viçosa: Ed. UFV, 2010. 269 p.

BARBOSA, O. R.; BOZA, P. R.; SANTOS, G. T. DOS; SAKAGUSHI, E. S.; RIBAS, N. P. $<$ b $>$ Efeitos da sombra e da aspersão de água na produção de leite de vacas da raça Holandesa durante o verão</b\&gt; - DOI: 10.4025/actascianimsci.v26i1.1961. Acta Scientiarum. Animal Sciences, v. 26, n. 1, p. 115-122, 10 abr. 2008.

BOHMANOVA, J.; MISZTAL, I.; COLE, J. B. Índices de Temperatura e Umidade como Indicadores de Perdas na Produção de Leite Por Estresse Térmico Journal of Dairy Science, $v$. 90, n. 4, p. 1947-1956, abr. 2007. https://doi.org/10.3168/jds.2006-513.

BRETTAS, P. K. M. Escolha do índice de estresse térmico e da origem dos dados do ambiente térmico para avaliar o estresse por calor em bovinos leiteiros em ambiente tropical. 2015. 92 f. Dissertação (Mestrado) - Curso de Medicina Veterinaria, Experimental, Universidade Federal de Uberlândia, Uberlandia, 2017. Cap. 3.

CUNHA, P. G. V.. Benefícios do cooperativismo aos produtores de leite: visão geral. 2020. 63 f. Monografia (Especialização) - Curso de Administração, Facer Faculdade de Ceres, CeresGoiás, 2020. Cap. 10. 
CERQUEIRA, J. O. L. Avaliação de Bem-Estar Animal em Bovinos de Leite na Região

Norte de Portugal. 2010. 342 f. Tese (Doutorado) - Curso de Medicina Veterinaria, Experimental, Instituto de Ciências Biomédicas Abel Salazar da Universidade do Porto, Porto, 2013. Cap. 7.

CONCEIÇÃO, M.N. Avaliação da Influência do sombreamento Artificial no Desenvolvimento de Novilhas Leiteiras em Pastagens. 2005. 138 f. Tese (Doutorado) - Curso de Engenharia Agronômica, Experimental, Universidade de São Paulo- Escola Superior de Agricultura "Luz de Queiroz", Piracicaba, 2008. Cap. 1.

DALTRO, A. M. Efeito do Estresse Térmico na Produção de Vacas Leiteiras. 2018. $31 \mathrm{f}$. TCC (Graduação) - Curso de Zootecnia, Universidade Federal do Pampa, Dom Pedrito, 2018. Cap. 13.

DOMingOS, H. G. T., et al. Influência do Sombreamento e Aspersão de Água Sobre a Produção de Leite e Respostas Fisiológicas de Vacas Leiteiras, Londrina-PR, v. 6, n. 9, p. 114, 14 set. 2012. Semestral.

FERREIRA, I. C., et al. Conforto Térmico em Bovinos Leiteiros a Pasto. Infoteca-E Repositório de Informação Tecnológica da Embrapa, Planaltina - Df, v. 3, n. 5,p.149,15mar.2017.Semestral.Disponível:http://www.infoteca.cnptia.embrapa.br/infoteca/handle/doc/ 1092310.. Acesso em: 8 jul. 2019.

MATARAZZO, S. V. Eficiência do sistema de resfriamento adibático evaporativo em confinamento do tipo freestall para vacas em lactação. 2004. 63 f. Tese (Doutorado) - Curso de Agronomia, Escola Superior de Agricultura Luiz de Queiroz, Jaboticabal -Sp, 2004. Cap. 11. Disponível em: http://www.sbea.org.br.. Acesso em: 22 mar. 2019.

ROSA, M. S. Estresse Térmico na produção de leite de bovinos leiteiros de raça girolando utilizando modelo de regressão aleatório. 2019. 66 f. Dissertação (Mestrado) - Curso de Zootecnia, Universidade Federal do Rio Grande do Sul. Faculdade de Agronomia, Rio Grande do Sul-Rs, 2019. Cap. 8. Disponível em: http://hdl.handle.net/10183/197815 Acesso em: 7 set. 2019.

SILVA, I. J. O., et al. Efeitos da Climatização do Curral de Espera na Produção de Leite de vacas Holandesas . R. Bras. Zootec. [online]. 2002, vol.31, n.5, pp.2036-2042. ISSN 18069290. https://doi.org/10.1590/S1516-35982002000800019.

VITOR. N. O.; BITTAR, D Y. Analise do conforto térmico e sua influência na produção e qualidade do leite em ambiente de domínio de cerrado. Pubvet Medicina Veterinária e Zootecnia, Goianésia, v. 12, n. 4, p. 1-6, 04 abr. 2018. Semestral. 